九州大学学術情報リポジトリ

Kyushu University Institutional Repository

\title{
Coactive neuro-fuzzy modelling for colour recipe prediction
}

Mizutani, Eiji

Informations Systems Dept., Kansai Paint Co.,Ltd.

Jyh-Shing R. Jang

Department of Computer Science, National Tsing Hua University

Nishio, Kenichi

Video Technology Center, Sony Corp.

高木，英行

Department of Acoustic Design, Kyushu Institute of Design

他

ht tp://hdl. hand le. net/2324/4485874

出版情報: Proceedings of 1995 IEEE International Conference on Evolutionary Computation. 5, pp. 2252-2257, 1995-11-27. IEEE

バージョン :

権利関係 : 


\title{
Coactive Neuro-Fuzzy Modeling for Color Recipe Prediction
}

\author{
Eiji Mizutani ${ }^{1}$, Jyh-Shing R. Jang ${ }^{2}$, Kenichi Nishio ${ }^{3}$ \\ eiji@joho.kansai.co.jp, jang@cs.nthu.edu.tw, nishio@cv.sony.co.jp \\ Hideyuki Takagi ${ }^{4}$, and David M. Auslander ${ }^{5}$ \\ takagi@kyushu-id.ac.jp, dma@euler.berkeley.edu \\ 1) Information Systems Dept., Kansai Paint Co.,Ltd, 4-3-6 Fushimi Chuo, Osaka 541 JAPAN \\ 3) Video Technology Center, Sony Corp., 5-9-12 Kitashinagawa Shinagawa, Tokyo 141, JAPAN \\ 4) Acoustic Design Dept., Kyushu Institute of Design, 4-9-1 Shiobaru,Minami-ku, Fukuoka 815, JAPAN \\ 5) Dept.of Mechanical Engineering, University of California at Berkeley, CA 94720, USA
}

2) Dept. of Computer Science, National Tsing Hua University, Hsinchu, Taiwan

\begin{abstract}
We explore neuro-fuzzy approaches to computerized color recipe prediction, which relates surface spectral reflectance of a target color to several colorant proportions. The approaches are expressed within the framework of CANFIS (CoActive Neuro-Fuzzy Inference System) where both Neural Networks (NNs) and Fuzzy Systems (FSs) play active roles together in pursuit of a given task.

To find an ideal adaptive model for this problem, we have investigated a variety of structures; they feature knowledge-embedded architectures and an adaptive FS, which serves to determine color selection. They have enormous potential for augmenting prediction capability.
\end{abstract}

\section{Introduction}

Colors enliven our daily lives. We often need a specific color exactly matched with our favorite color. Yet our color perception is very sensitive. Hence, in the color industry, it is important to predict the proportions of several colorants (or color pigments) needed to produce the same color as a given target color. The color matching must be done very well to satisfy severe requirements. In a practical situation, it is necessary to examine the color match in daylight as well as in artificial light. It is actually an arduous task. The prediction process involves choice of necessary colorants, and requires high precision of their proportions.

Simple NN approaches to color recipe prediction have been discussed well $[1,10,8]$. Under practical environments, we sometimes encounter severe standards, which may be hard to meet by employing an NN alone. In the past few years, Neuro-Fuzzy models have been much studied; the literature promises far wider industrial applications. We contend that another approach such as fuzzy modeling must complement simple NNs to enhance their overall performance.

\section{CANFIS Concept}

CANFIS has expanded the concept of a singleoutput system, ANFIS [5](Adaptive Network-based Fuzzy Inference System), which realizes fuzzy inferencing in the network-layered representation. Fig-

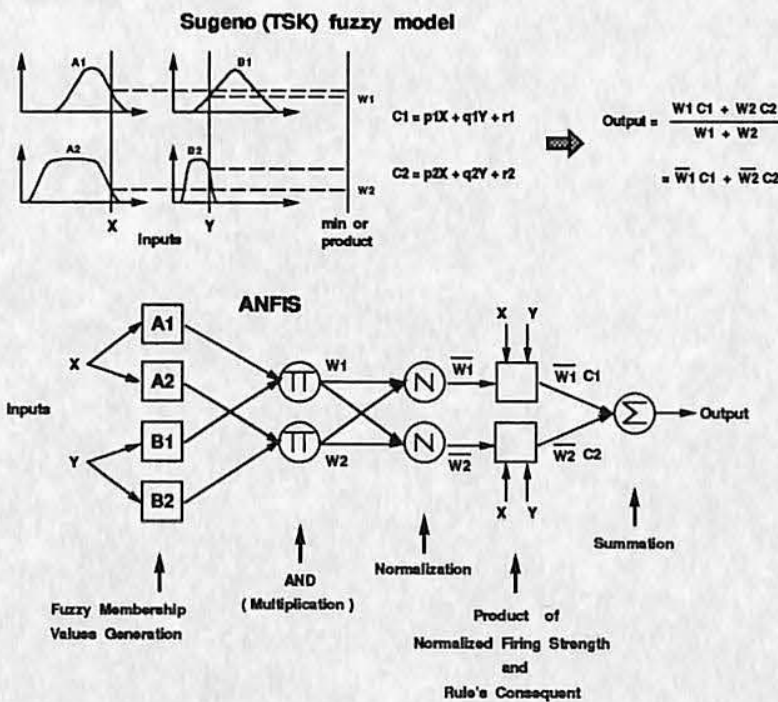

Fig. 1: First-order Sugeno (TSK) fuzzy model (above) and a corresponding ANFIS structure (below).

ure 1 illustrates a comparison between the TSK fuzzy inference system [11] and a corresponding ANFIS to perform the following fuzzy rules (for further details, refer to $[5,6])$ :

$$
\begin{aligned}
& \text { Rule1 If } \mathrm{X} \text { is } A_{1} \text { and } \mathrm{Y} \text { is } B_{1} \text {, } \\
& \text { then } \quad C_{1}=p_{1} \mathrm{X}+q_{1} \mathrm{Y}+r_{1} \text {. } \\
& \text { Rule2 If } \mathrm{X} \text { is } A_{2} \text { and } \mathrm{Y} \text { is } B_{2} \text {, } \\
& \text { then } C_{2}=p_{2} \mathrm{X}+q_{2} \mathrm{Y}+r_{2} \text {. }
\end{aligned}
$$

CANFIS, a generalized ANFIS, can produce 


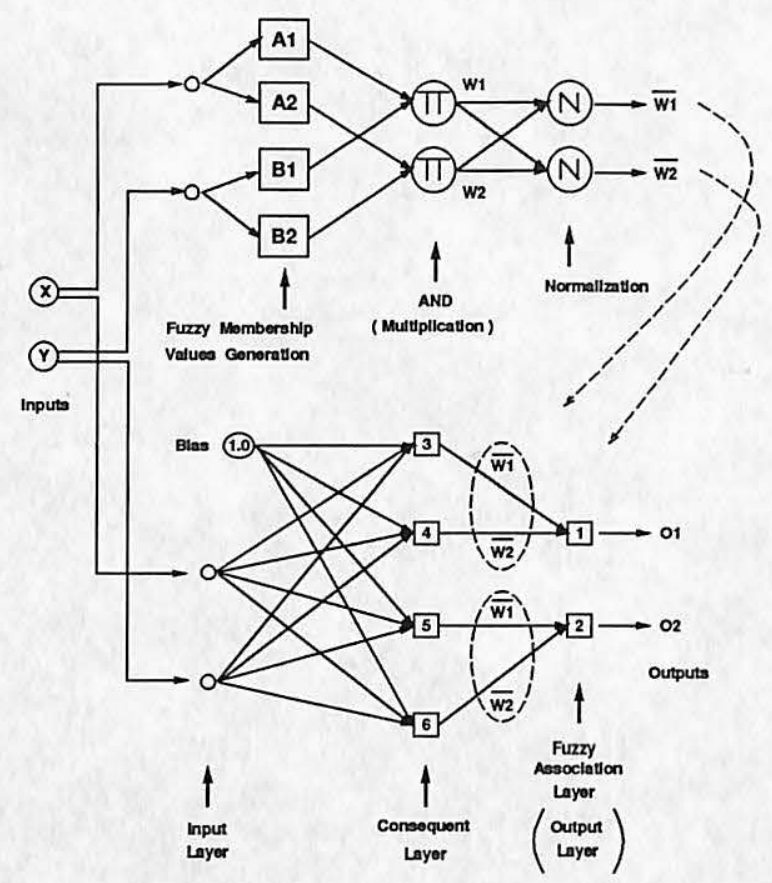

Fig. 2: CANFIS: multiple-output ANFIS.

multiple outputs and take advantage of nonlinear rule formations [9]. Figure 2 describes a simple CANFIS model to produce multiple outputs. This model shares antecedent parts unlike MANFIS (Multiple ANFISs) model wherein several ANFISs are just juxtaposed.

\subsection{Rule Formation}

Suppose we have a sigmoidal function as a neuron function in the consequent layer such as Functions $3,4,5$, and 6 in Figure 2. Then we have a nonlinear consequent, $C_{n o n}$ :

$$
C_{n o n}=\frac{1}{1+\exp \left[-\left(p_{1} X+q_{1} Y+r_{1}\right)\right]} .
$$

In this case, we have a sigmoidal rule.

Furthermore, when we introduce an NN in a rule construction, we have a rather complicated structure as illustrated in Figure 3 (above). Moreover, when such neural consequents are entwined; that is, when two neural consequents, "Neural Rule ${ }_{1}$ " and "Neural Rule 3 " are combined to form one neural rule (Local Expert $N N_{1}$ ), and "Neural Rule ${ }_{2}$ " and "Neural Rule 4 " are fused into another neural rule (Local Expert $\mathrm{NN}_{2}$ ), we have a construction similar to a typical modular network as illustrated in Figure 3 (below) where the outputs of two local expert NNs are mediated by an integrating unit (typically a gating network) $[4,3,7]$. In another perspective, a task is split up among several neural rules or NNs. Thus, the entire model may be able to overcome the individual limitation of any one $\mathrm{NN}$; so the average

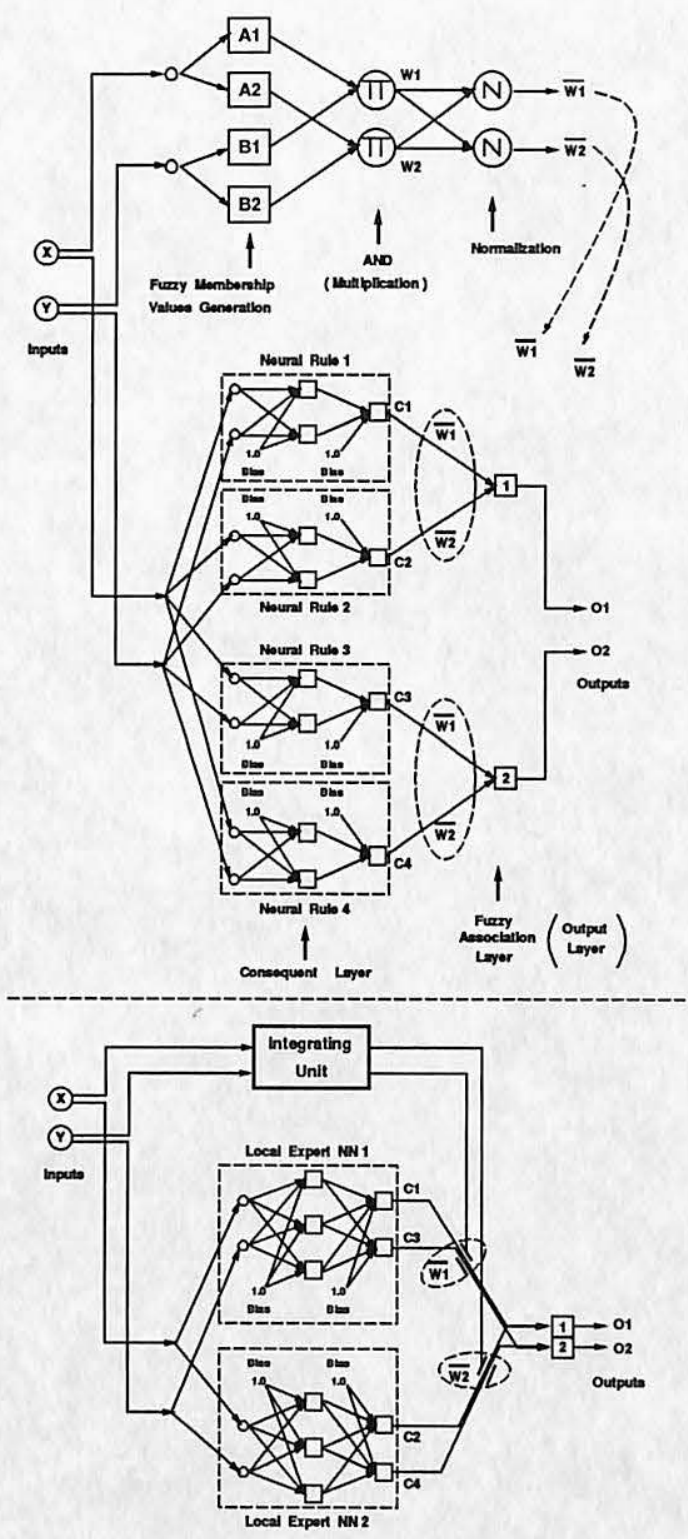

Fig. 3: CANFIS for multiple outputs with four neural rules (above), and a comparable typical modular network (below).

load of each NN may be reduced. Here the idea is task decomposition. (CANFIS with neural rules can be regarded as an introduction of fuzzy concept into a modular network.)

We shall show how CANFIS can be generalized for application to color recipe prediction in the next section.

\section{CANFIS modeling for Color Recipe Prediction}

The training data consist of 1,446 Munsell color chips and the checking data of 302 standard paint color chips of the Japan Paint Manufacturers Association. Both data sets were sampled from surface spectral reflectance at 16 points, ranging from 

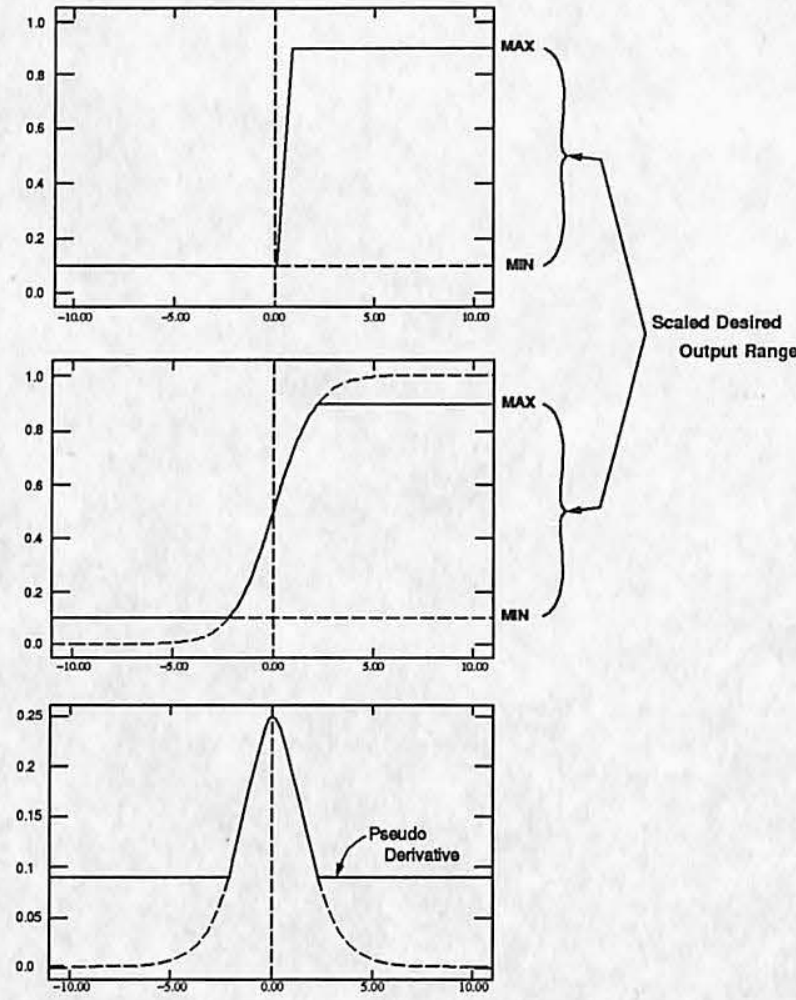

Fig. 4: A truncation filter (top), and a modified sigmoida function (middle) compared with its derivative (bottom) when MAX is 0.9 and MIN is 0.1 . By contrast, dashed lines show a normal sigmoidal logistic function.

$400 \mathrm{~nm}$ to $700 \mathrm{~nm}$ in wavelength (20-nm intervals). We have such 16 spectral inputs and 10 colorant outputs. In our color recipe prediction, we must select several color pigments out of the ten colorant candidates, and determine their proportions.

\subsection{Neuron Functions}

In our data sets, the average number of color pigments to produce any color was about four. This means six of the ten final outputs should be zero. In addition, we sometimes need to predict precise proportions with enough precision to specify levels such as $0.01 \%$. It is an important concern in color recipe prediction to specify such extremities of the output range [1]. To cope with it, as an improvement of single NN approaches, we have employed modified sigmoidal functions, $f_{\text {mod }}$, and truncation filter functions, $f_{t r c}$, as neuron functions for the fuzzy association layer or the final output layer $[7,8]$ such as Functions 1 and 2 in Figure 2; they are defined below:

$$
\begin{gathered}
f_{\text {trc }}(x)= \begin{cases}\text { MIN } & \text { if } \mathrm{x} \leq \mathrm{MIN} \\
\text { MAX } & \text { if } \mathrm{x} \geq \text { MAX } \\
x & \text { otherwise }\end{cases} \\
f_{\text {mod }}(x)= \begin{cases}\text { MIN } & \text { if } \mathrm{f}(\mathrm{x}) \leq \mathrm{MIN} \\
\text { MAX } & \text { if } \mathrm{f}(\mathrm{x}) \geq \mathrm{MAX} \\
\mathrm{f}(\mathrm{x}) & \text { otherwise }\end{cases}
\end{gathered}
$$

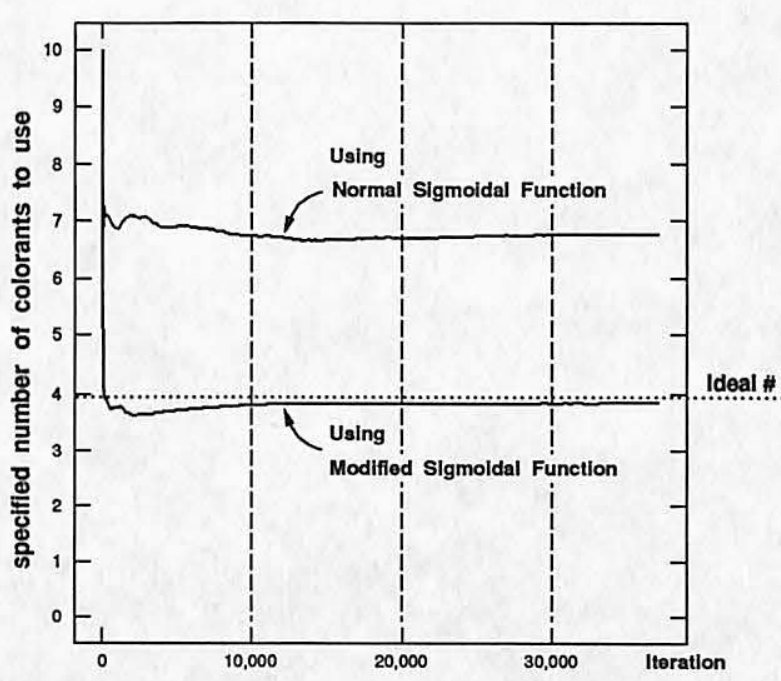

Fig. 5: Number of necessary pigments predicted by an NN with the normal sigmoidal logistic functions and an NN with the modified sigmoidal functions. The desired number of pigments is about four in this experiment.

where $f(x)$ is the normal sigmoidal logistic function given by

$$
f(x)=\frac{1}{1+\exp (-x)},
$$

and we set MIN to 0.1 , and MAX to 0.9 . To draw advantage of these functions, we need to scale the range of desired outputs to the range [MIN,MAX] (detailed in [8]). Notice that in the calculation of the derivative of the modified sigmoidal function, we introduced a pseudo derivative to keep a certain amount of derivative. Fahlman also suggested a similar method whereby a small magic constant (0.1) was simply added to the calculation of the derivative [2]. These neuron functions are depicted in Figure 4.

In the preliminary experiments using a single NN, the effects can be seen clearly in Figure 5 . The NN with normal sigmoidal functions, $N N_{\text {normal }}$, tended to specify use of more pigments than necessary; it averaged almost seven specified pigments. On the other hand, the NN with modified sigmoidal functions, $N N_{\text {mod }}$, shows the predicted number of pigments asymptotically approached the ideal number of pigments as iteration progressed. The outputs were further processed to eliminate redundant pigments at the MIN value. The truncation filter has an effect similar to the modified sigmoidal function in eliminating redundant colorants. This truncation function is used in CANFIS models, which we shall discuss later. Again, note that we use these functions only in the fuzzy association layer. 


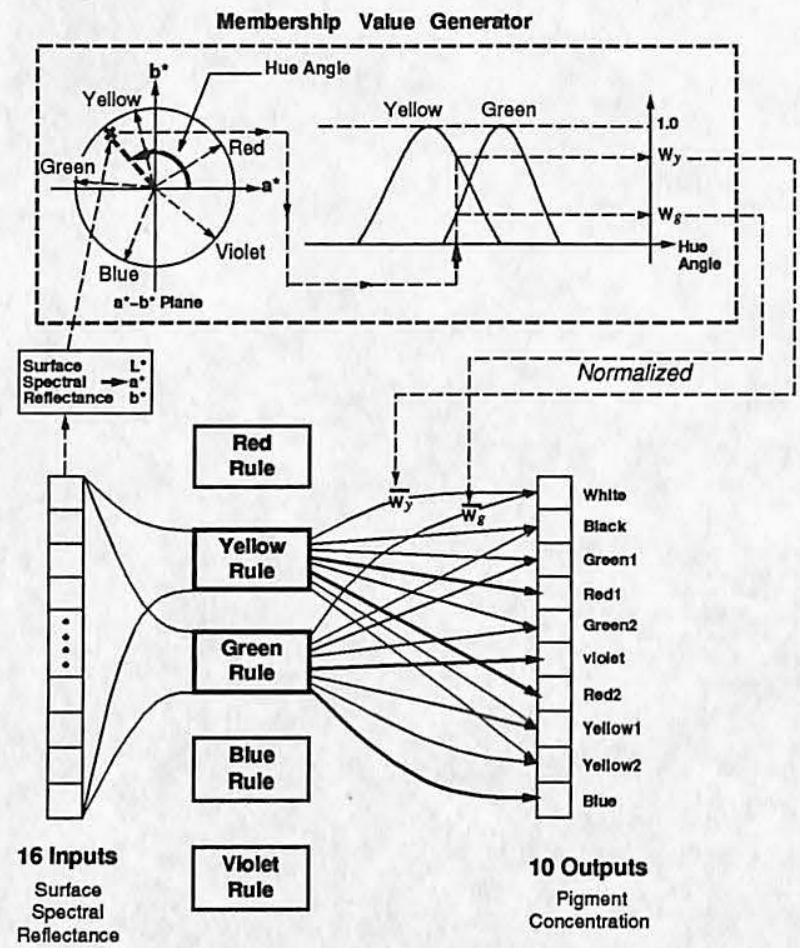

Fig. 6: A CANFIS model with five rules for color recipe prediction. MFs are set up for the hue aspect alone.

\subsection{Fuzzy Partitionings}

In fuzzy modeling, it is of importance to determine a reasonable number of MFs to maintain appropriate linguistic meanings. In all simulation examples of ANFIS [5], MFs were set up for all inputs using grid partitions, but here is a question; is it necessary to set up MFs for all 16 spectral inputs? In such a case, we must have the following 16 fuzzy rules:

Rule 1: If $X_{1}$ (at $400 \mathrm{~nm}$ ) is $A_{1}$, then $C_{1}$, Rule 2: If $X_{2}$ (at $\left.420 \mathrm{~nm}\right)$ is $A_{2}$, then $C_{2}$,

Rule 16: If $X_{16}($ at $700 \mathrm{~nm})$ is $A_{16}, \quad$ then $C_{16}$. where $A_{i}$ denotes fuzzy linguistic labels. (Note that the visible range of color spectrum is $400 \mathrm{~nm}$ $700 \mathrm{~nm}$.) These rules may not make sense since we do not have such explicit knowledge per wavelength. Without explicit domain-knowledge, adaptive learning mechanisms enable ANFIS / CANFIS to cause a buildup of fuzzy rules automatically. But if the initial setup of MFs has no meanings, it must be futile to extract fuzzy rules from a fuzzy logic point of view. Blindly applying fuzzy MFs to all scalar inputs may turn out to be meaningless.

To keep meaningful fuzzy rules, the number of MFs should be carefully determined so that those fuzzy rules can be specified. Fortunately, there is a formula to transform surface spectra of color to perceptual attributes, 'lightness,' 'hue,' and 'chroma' [12]. These three values must be more

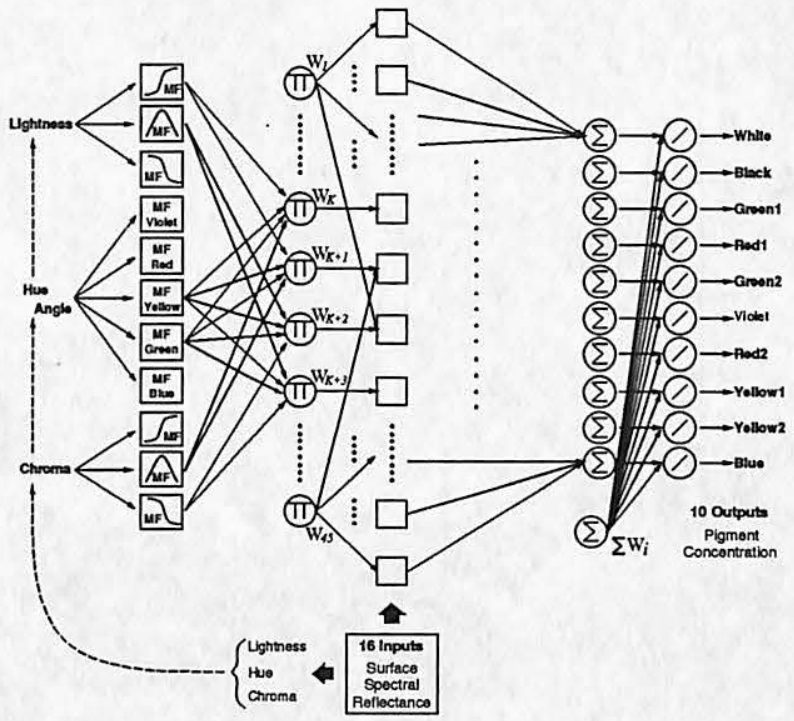

Fig. 7: A CANFIS model with 45 rules for color recipe prediction. MFs are set up for the three color attributes, 'lightness,' 'hue,' and 'chroma.'

suitable for treating color in a linguistically meaningful way than the 16 spectral values. Hence, we use them as three inputs for MFs but not 16 inputs of surface spectral reflectance. When we invert the 3-D partitionings in the color-attribute space to the 16-D of spectral inputs, certain complicated partitions must be constructed in the 16-D space.

Notice that an NN with 16 spectral inputs did a better job than an NN with three inputs of 'lightness,' 'hue,' and 'chroma.' Thus, as illustrated in Figures 6 and 7, five color neural rules have 16 inputs (color spectra) whereas MFs have those three inputs (color attributes).

\subsection{Knowledge-embedded structures}

Adaptive fuzzy membership functions (MFs) specify the degree of membership of five color regions (red, yellow, green, blue, violet) according to perceptual attributes of color, 'lightness,' 'hue,' and 'chroma' [12]. They determine what weight should be assigned to each rule's output in order to produce a final output. We have applied the colorist's knowledge to the CANFIS architecture so that several connections can be pruned. For example, a green rule (weighted by a green MF) has no effect on the proportions of red pigments because of the green-red complementary color relationship. After the pruning process, each color rule has fewer output units than the ten final outputs; Figure 6 pictures this idea, which can be viewed as a variation of a modular network in Figure 3 (below). Again, in this experiment, six of the ten final outputs should be zero because the desired number of color pigments to produce any color is about four. Reducing the number of zero outputs 
Table: 1: Detail descriptions of the four representative CANFIS models. The bell-shaped MFs and the modified bell MFs are defined in Equation (4) and Equation (5), respectively.

\begin{tabular}{l|l}
\hline (a) & $\begin{array}{l}\text { CANFIS with } 5 \text { sigmoidal rules (see Equation (1)) } \\
\text { with pruned connections } \\
5 \text { bell-shaped MFs are set up for hue angle alone } \\
\text { (i.e., } 5 \text { rules are for five color regions) }\end{array}$ \\
\hline (b) & $\begin{array}{l}\text { CANFIS with } 45 \text { linear rules as in Figure } 7 \\
\text { with NO pruned connections } \\
3 \text { bell-shaped MFs are set up for lightness } \\
3 \text { bell-shaped MFs are set up for chroma }\end{array}$ \\
& $\begin{array}{l}5 \text { bell-shaped MFs are set up for hue angle } \\
\text { (c) }\end{array}$ \\
& $\begin{array}{l}\text { CANFIS with } 5 \text { neural rules as in Figure 3(below) } \\
\text { with } \text { modified bell MFs are set up for hue angle alone } \\
5 \text { neural rules have the same model size } \\
\text { (i.e., Each neural rule has } 22 \text { hidden units) }\end{array}$ \\
\hline (d) & $\begin{array}{l}\text { CANFIS with } 5 \text { neural rules as shown in Figure } 6 \\
\text { with pruned connections } \\
5 \text { modified bell MFs are set up for hue angle alone } \\
\text { Each neural rule is heuristically optimized; } \\
\text { i.e., their model sizes are specified in Table 2 }\end{array}$
\end{tabular}

can possibly have a positive impact on the inputoutput mappings.

\subsection{Fuzzy Membership Functions}

Fuzzy membership functions play a role of an integrating unit; we consider the following bell-shaped MFs:

$$
\mu(x)=\frac{1}{1+\left|\frac{x-c}{a}\right|^{2 b}},
$$

or

$$
\mu_{\text {mod }}(x)=\max \left\{\frac{2}{1+\left|\frac{x-c}{a}\right|^{2 b}}-1,0\right\},
$$

where $\{a, b, c\}$ is an adjustable parameter set. The latter definition is a modified bell MF, which is just upper half part of the original bell-shaped MF.

In structural terms of CANFIS with neural rules, it has many adjustable parameters compared with single NN models (such as $N N_{\text {normal }}$ and $N N_{\text {mod }}$ ). To accelerate training, we can employ the modified bell MFs defined in Equation (5) to control the number of firing rules; the functions are instrumental for this color recipe prediction because it may not be necessary to use more than two color rules at the same time. More specifically, when the target color is in a region between green and yellow; that is, when both the "yellow rule" and the "green rule" are fired, the neighboring "red rule" and "blue rule," are not necessarily fired because of the yellow-blue and green-red complementary color relationships. Several weight-updating procedures for unnecessary or inactive rules can then be skipped when iterative training procedures are employed.
Table: 2: An optimal model structure of five color NNs and their initial number of training / test data. Note that each neural rule has fewer outputs than the ten final outputs.

\begin{tabular}{|c|c|c|c|}
\hline $\begin{array}{c}\text { Five Color } \\
\text { Neural Rule }\end{array}$ & Model Size & $\begin{array}{c}\text { Training } \\
\text { Data }\end{array}$ & $\begin{array}{c}\text { Checking } \\
\text { Data }\end{array}$ \\
\hline Rule $_{\text {Red }}$ & $16 \times 16 \times 16 \times 8$ & 650 & 138 \\
\hline Rule $_{\text {Y llow }}$ & $16 \times 16 \times 17 \times 8$ & 707 & 200 \\
\hline Rule $_{\text {Green }}$ & $16 \times 21 \times 7$ & 521 & 105 \\
\hline Rule $_{\text {Blue }}$ & $16 \times 15 \times 8$ & 363 & 65 \\
\hline Rule Violet & $16 \times 17 \times 6$ & 409 & 48 \\
\hline
\end{tabular}

\section{Simulation}

We have tested various CANFIS models; because of the space limitation, we show the several representative CANFIS models as shown in Table 1. Table 3 shows performance comparison of those CANFIS models as well as single NN models: $N N_{\text {normal }}$ and $N N_{\text {mod }} ; N N_{\text {mod }}$ is an improved $N N$ model with the modified sigmoidal function.

There must be many possible optimal rule formations; Table 2 shows one of them, which we found through trial and error implementation, using a model depicted in Figure 6. Each color neural rule (or local color NN) has a different model size because different number of data goes into each neural rule; those initial number of data is also shown in Table 2. Notice that each neural rule has fewer outputs than the ten final outputs because of the pruning procedure discussed in Section 3.3. By contrast, we also tested a CANFIS in which all five neural rules had the same model size.

Through the preliminary CANFIS modeling, we found that CANFIS with the truncation filter functions defined in Equation (2) at the fuzzy association layer worked better than CANFIS with the identity functions. We did not present any results about this, but the results were more or less the same as those of $N N_{\text {normal }}$ and $N N_{\text {mod }}$ with respect to the specified number of colorants to use; that is, CANFIS with the identity functions at the fuzzy association layer tended to specify use of more colorants than necessary. We verified the truncation filter functions at the fuzzy association layer surely helped in eliminating redundant pigments.

We used a priori knowledge to prune some connections as discussed in Section 3.3. When we employed such pruned structures, we did obtain at least a few percent improvement in terms of pigment errors. (Note that we did not present any comparisons about this finding in Table 3 .)

\section{Discussion}

We have applied a variety of CANFIS structures as a touchstone to the color recipe prediction for discovering what CANFIS model belongs to the truly 
Table: 3: Performance comparison between single NN models: $N N_{\text {normal }}$ and $N N_{\text {mod }}$, and four representative CANFIS models. Table 1 details the four CANFIS models.

\begin{tabular}{|c|c|c|c|c|c|c|c|c|}
\hline & \multicolumn{3}{|c|}{ \# of membership functions } & \multicolumn{2}{|c|}{ Rule } & \multirow{2}{*}{$\begin{array}{c}\text { Checking } \\
\text { error }\left(\times 10^{-2}\right)\end{array}$} & \multirow{2}{*}{$\begin{array}{l}\text { Specified \# } \\
\text { of pigments }\end{array}$} & \multirow{2}{*}{$\begin{array}{c}\text { Parameter } \\
\text { number }\end{array}$} \\
\hline & hue & lightness & chroma & no. & formation & & & \\
\hline CANFIS (a) & 5 & 0 & 0 & 5 & sigmoidal & 7.99 & 3.46 & 852 \\
\hline CANFIS (b) & 5 & 3 & 3 & 45 & linear & 2.59 & 3.76 & 7,683 \\
\hline CANFIS (c) & 5 & 0 & 0 & 5 & neural & 1.90 & 3.85 & 3,035 \\
\hline CANFIS (d) & 5 & 0 & 0 & 5 & neural & 1.41 & 4.00 & 2,691 \\
\hline$N N_{\text {normal }}$ & - & - & - & 1 & neural & 2.62 & 6.66 & 925 \\
\hline$N N_{\text {mod }}$ & - & - & - & 1 & neural & 2.03 & 3.90 & 925 \\
\hline
\end{tabular}

excellent class; at this stage, the CANFIS model (d) presented the best performance as in Table 3.

CANFIS with 45 linear rules has much more modifiable parameters than other CANFIS models (see Table 3). Accordingly, it required much more computation time than other CANFIS models; so we did not successfully optimize parameter setups for CANFIS with 45 linear rules in heuristic ways. Because of its huge construction, it seems difficult to introduce neural rules (or local color expert NNs) to CANFIS with $11 \mathrm{MFs}$ (or 45 rules); therefore, we did not test CANFIS with 45 neural rules. From a color science standpoint, however, it is a better idea to consider the three color attributes: 'lightness,' 'hue,' and 'chroma,' than to consider the 'hue' aspect alone. We thus must make an effort to upgrade such a CANFIS model with 11 MFs. At the same time, we should consider whether there is a more effective way to represent human visual sensitivity to color in the perceptual attribute space than the use of bell-shaped MFs. We may need to contrive a more sophisticated MF.

It was observed that modified bell MFs defined in Equation (5) were useful in accelerating learning because we used the iterative gradient decent (GD) backpropagation method. In the future work, we should apply to CANFIS models the previouslyproposed hybrid learning algorithm, which is based on a combination of the GD method and the leastsquares estimation method $[5,6]$.

\section{Conclusion}

We have demonstrated the strength of knowledgeembedded CANFIS models in the computer color recipe prediction.

By constructing MFs in the color-attribute space, the CANFIS models have realized meaningful and concise representations of colorists' knowledge. These encouraging results confirm that CANFIS offers superior performance and better learning capabilities than single NN models. But the results are not conclusive in determining most appropriate CANFIS architectures; we need to investigate more experimentations.
To explore the discussed concerns must be our next step, which may endow a breakthrough in understanding neuro-fuzzy modeling. We believe such efforts could pave the way for a new generation of CANFIS.

\section{References}

[1] Bishop, J.M., Bushnell, M.J., Westland, S., "Application of Neural Networks to Computer Recipe Prediction," Color Research and Application, Vol.16, No.1, pp.3-9, (1991)

[2] Fahlman, Scott. E., "Faster-Learning Variations on Back-Propagation: An Empirical Study," Proceedings of the 1988 Connectionist Models Summer School, (1988)

[3] Haykin, Simon, "Modular Networks," (chapter 12) in Neural Networks: a comprehensive foundation, Macmillan College Publishing Company, Inc. (1994).

[4] Jacobs, R.A., Jordan, M.I., Nowlan, S.J., Hinton, G.E., "Adaptive mixtures of local experts," Neural Computation 3, pp.79-87 (1991)

[5] Jang, J.-S. Roger., "ANFIS: Adaptive Network-based Fuzzy Inference Systems," IEEE Trans. on Systems, Man and Cybernetics, vol.23. No.3, pp.665-685 (1993)

[6] Jang, J.-S. Roger, Sun, C.-T., and Mizutani, E., NeuroFuzzy Modeling and Soft Computing, Prentice Hall (in press)

[7] Mizutani, E., Jang, J.-S. R., Nishio, K., Takagi, H., Auslander, D.A., "Coactive Neural Networks with Adjustable Fuzzy Membership Functions and Their Applications," Int'l Conf. on Fuzzy Logic, Neural Nets, and Soft Computing (IIZUKA '94)

[8] Mizutani, E., Takagi, H., Auslander, D.M., "A Cooperative system based on soft computing methods to realize higher precision of computer color recipe prediction," SPIE's Int'l Symposium on OE/Aerospace Sensing and Dual Use Photonics (1995)

[9] Mizutani, E., and Jang, J.-S. R., "Coactive Neural Fuzzy Modeling," IEEE Int'l Conf. on NN (ICNN'95), (will appear)

[10] Spehl, J., Wolker, M., Pelzl, J., "Application of Backpropagation Nets for Color Recipe Prediction as a Nonlinear Approximation Problem," IEEE Int'l Conf. on NN (ICNN'94), pp.3336-3341 (June, 1994)

[11] Takagi, T. and Sugeno M., "Fuzzy identification of systems and its application to modeling and control," IEEE Trans. on Systems, Man and Cybernetics, vol.15, pp.116-132 (1985)

[12] Wyszecki, G. and Stiles, W.S., "Color science: concepts and methods, quantitative data and formulae," New York, NY: Wiley, 2nd ed. (1982) 